\title{
Trapped Placenta and Conceptus in Uterine Sacculation: Two Rare Case Reports
}

\author{
MS Kokila, H Indira, Akhilesh Dutta Dwivedi, L Dwarakinath
}

\begin{abstract}
Uterine sacculation is a very rare pregnancy complication having an incidence of about one in 3000 pregnancies. It is more commonly associated with retroverted uterus. Isolated sacculation either congenital or acquired is very rare. Without timely intervention, this can cause miscarriage, intrauterine fetal death, preterm delivery, uterine rupture, adherent/retained placenta and postpartum hemorrhage. Retained placenta is a common complication of congenital sacculation. Acquired sacculation occurs secondary to uterine surgeries, like uterine curettage, hysterotomy, cesarean section or following misoprost use. A rare case of retained placenta in the congenital uterine sacculation, first diagnosed during laparotomy done for failed manual removal of placenta, which was removed by hysterotomy. Another case with failed induction of abortion for intrauterine fetal demise and threatened rupture of acquired sacculation are reported.

Awareness and early diagnosis of uterine sacculation allows clinicians to be prepared for emergency situations and better manage complications, to limit fetomaternal morbidity and mortality.
\end{abstract}

Abbreviations: IUGR: Intrauterine growth restriction; IUFD: Intrauterine fetal death.

Keywords: Uterine sacculation, R etained placenta, Misoprost, Hysterotomy.

How to cite this article: Kokila MS, Indira $H$, Dwivedi AD, Dwarakinath L. J South Asian Feder Obst Gynae 2013;5(2): 89-90.

\section{Source of support $\mathrm{Nil}$ \\ Conflict of interest: None declared}

\section{INTRODUCTION}

Isolated uterine sacculation (diverticulum) is a very rare complication. Sacculation occurs in about one in 3000 pregnancies with retroverted uterus. ${ }^{1}$ The condition has been defined as a transitory pouch or saclike structure developing from a portion of the gravid uterus and containing all the layers of the uterus. This can cause spontaneous miscarriage, intrauterine fetal death, uterine rupture, preterm delivery, abnormally adherent retained placenta and postpartum hemorrhage. ${ }^{1}$ T wo cases of uterine sacculation with different presentation, where hysterotomy was needed to remove retained placenta and conceptus are reported.

\section{CASE REPORTS}

\section{Case 1}

A primipara presented with retained placenta and hemorrhage 4 hours after vaginal delivery of a live term intrauterine growth restriction (IUGR) baby, with no past history of any uterine surgery or curettage. A ntenatal ultrasound had reveal ed excess placental calcification.
On examination, she was in shock. U terus was 24 weeks size, well contracted and, on examination, a bulge was noted in left uterine cornua. Speculum examination revealed about $500 \mathrm{gm}$ of blood clots and cord coming out of partially opened cervix. Placenta was inaccessible. In view of active hemorrhage and to hasten placental separation, oxytocin was started as infusion and also through umbilical vein. Manual removal of placenta was attempted under general anesthesia and failed, as cervix did not open even after maximum dose of muscle relaxants and deepening plane of anesthesia. In view of continued excessive bleeding and placental inaccessibility and cornual bulge being clearly felt, decision of hysterotomy was made. On laparotomy, uterine sacculation was seen in the left cornua, superomedial to the ovarian ligament, as a bluish red bulge of about $5 \times 8 \mathrm{~cm}$, with dilated and tortuous vessels on its surface (Figs 1 and 2). A t hysterotomy, placenta was found partially separated and trapped in the sacculation and removed piecemeal. Placenta was small (250 gm) with multiple calcifications and infarcts. U terine exploration revealed, extremely thinned out wall of sacculation. A fter placental removal, uterus was well contracted and hemostasis achieved. Postoperative period was uneventful. She was called for followup evaluation after 6 weeks.

\section{Case 2}

A 30-year-old multigravida presented with 18 weeks of gestation and ruptured membranes. She had history of spontaneous expulsion of dead fetus in first and cesarean sections in subsequent two pregnancies.

On abdominal examination, uterus was of 18 w eeks in size, relaxed without scar tenderness. Intrauterine fetal death (IUFD) with ruptured membranes and cord prolapse was diagnosed. Cervix was uneffaced admitting only tip of the finger.

She was induced with dinoprostone gel followed by two doses of misoprostol (100 mcg) at 6 hours interval. But failed

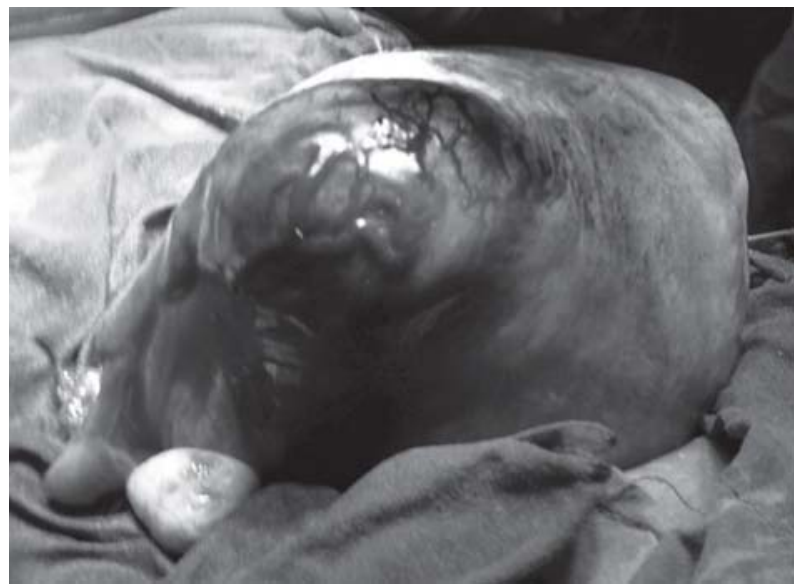

Fig. 1: Uterine sacculation in left uterine cornua, superomedial to ovarian ligament with dilated tortuous vessels on its surface 


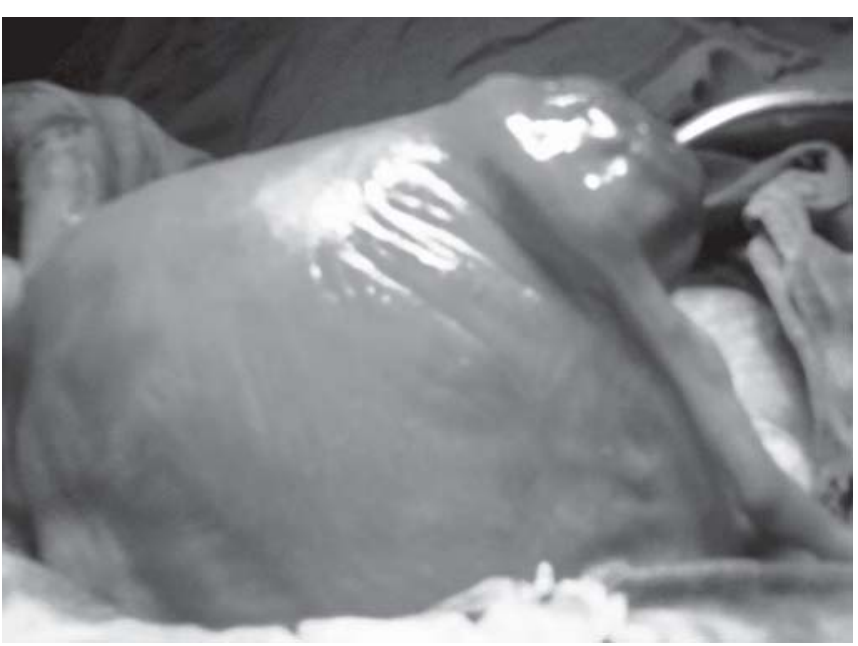

Fig. 2: Anterior view of sacculation

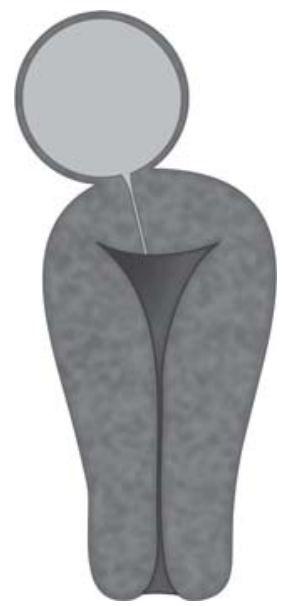

Fig. 3: Diagrammatic representation of sacculation

to abort even after having uterine contractions. In view of previous two cesarean sections and failed induction, emergency hysterotomy was performed and sacculation with extremely thinned out wall was seen in left cornua. Removal of trapped dead conceptus in the sac and tubectomy was done. Postoperative course was uneventful.

\section{DISCUSSION}

A true (congenital) sacculation is very rare. The sacculation is composed of all three layers of the uterine corpus with an excessively thinned out layer of myometrium that is continuous with the normal myometrium of the corpus (Fig. 3). Placental implantation and trophoblastic destruction of the uterine wall was considered to cause, saccular distention (U mezaki et al) ${ }^{2}$ or ballooning and excessive vascularity. It is not rudimentary horn as it bears no anatomic relationship to the cornual structures. Regions of fibrosis cause poor decidualization, placental insufficiency, IU GR and adherent retained placenta. A s uterus contracts postpartum and opening of sacculation into uterine cavity collapse, placenta becomes retained as in case 1. For the same reason placenta had to be removed piecemeal. Failure of cervical dilatation may be due to inadvertent use of methylergometrine before placental removal. It is impossible to remove the retained placenta in the sac by usual methods of manual removal or curettage, so most of them end up having a laparotomy and hysterotomy. ${ }^{3}$ Hysterectomy should be considered if sacculation is accompanied by abnormal placental adherence or serious hemorrhage. For selective cases of adherent retained placenta without bleeding, conservative methods like uterine artery embolization and administration of methotrexate, may be tried, as in a case reported by Choung et al. ${ }^{4}$

A cquired sacculation can be rel ated to prior uterine surgery or misoprostol use as in case $2 .^{2}$ It is difficult to diagnose till it ruptures silently and prove to be lethal without timely intervention. U se of strong uterotonic in postcesarean pregnancy for IUFD should be done with great caution and not to be waited too long before considering hysterotomy. In this case, insistence with uterotonics would have certainly led to rupture of uterus with grave consequences.

A wareness and early diagnosis of uterine sacculation would allow clinicians to be prepared for emergency situations and to better manage the complications to limit fetomaternal morbidity and mortality.

\section{Synopsis}

Hysterotomy to remove trapped placenta or conceptus in uterine sacculation limit maternal morbidity and mortality.

\section{REFERENCES}

1. LeeSW, K im M Y, Y ang JH, M oon M H, Cho JY. Sonographic findings of uterine sacculation during pregnancy. Ultrasound in Obstetrics \& Gynecology 2008;32:595-597.

2. Schmidt AP, Glitz CL, Passos EP, A rbo E, Cunha-Filho JS. $H$ ysteroscopy and three-dimensional ultrasonography in uterine diverticulum diagnosis: A case report. A m J Obstet Gynecol 2004 Feb;190(2):561-562.

3. Song JE, Lee SY, Park ST, Park SH, J un HA, Lee KY. A case of trapped placenta in uterine sacculation after normal delivery. K orean J Obs and Gyne 2006;49(6):1338-1344.

4. Choung $M$, Rho JH, Son CU, N a W S, Lee BK, Song Y R, Ryu $\mathrm{J} H$, H wang IT, K im K H. A case of placenta increta in the uterine sacculation which was treated with conservative methods. K orean J Perinatol 2007;18(3):277-285.

\section{ABOUT THE AUTHORS \\ MS Kokila}

A ssociate Professor, Department of Obstetrics and Gynecology Sapthagiri Institute of M edical Sciences, B engaluru, Karnataka, India

CorrespondenceAddres Siddarameswara Nilaya, 4th Cross, Siddaganga Extension, Tumkur-572102, K arnataka, India, Phone: 08162260270 M obile: 09449793266, e-mail: kokisreeni@ gmail.com

\section{H Indira}

Assistant Professor, Department of Obstetrics and Gynecology, Sri Siddhartha M edical College, Tumkur, K arnataka, India

\section{Akhilesh Dutta Dwivedi}

Professor, Department of Obstetrics and Gynecology, Sri Siddhartha M edical College, Tumkur, Karnataka, India

\section{Dwarakinath}

A ssociate Professor, Department of Obstetrics and Gynecology, Sri Siddhartha M edical College, Tumkur, K arnataka, India 